\title{
Synthèse bibliographique sur le comportement de recherche de l'hôte chez la punaise de lit (Cimex lectularius) et applications dans le cadre de la lutte intégrée
}

\author{
Pauline Legrand, François Verheggen, Éric Haubruge, Frédéric Francis
}

Université de Liège - Gembloux Agro-Bio Tech. Unité de Recherche Agrobiochem. Laboratoire d'Entomologie fonctionnelle et évolutive. Passage des Déportés, 2. BE-5030 Gembloux (Belgique). E-mail : entomologie.gembloux@ulg.ac.be

Reçu le 7 juillet 2015, accepté le 16 décembre 2015.

Description du sujet. Cette étude analyse le comportement de recherche de l'hôte chez la punaise de lit, Cimex lectularius, en portant une attention particulière sur les stimuli émis par l'Homme et impliqués dans ce processus d'orientation. Elle met également en évidence les applications possibles en matière de lutte intégrée.

Littérature. La punaise de lit est un ectoparasite exclusivement hématophage étroitement lié à l'Homme depuis des millénaires. Lors de sa recherche alimentaire, cet insecte utilise des stimuli émis par son hôte pour s'orienter efficacement. Le dioxyde de carbone issu de la respiration attire davantage l'insecte que la chaleur. D'autres composés chimiques émis par l'Homme sont testés mais leur perception sensorielle et leur influence comportementale ne sont pas toujours analysées conjointement et présentent dans certains cas des résultats discordants.

Conclusions. Aujourd'hui, le dioxyde de carbone reste le leurre le plus efficace dans les pièges, bien qu'il soit moins attractif qu'un appât humain. Des pièges artisanaux à base de glace carbonique seraient même plus efficaces que certains pièges utilisant des mélanges complexes de composés chimiques. L'étude du processus d'attraction de la punaise de lit vis-à-vis de l'Homme reste à parfaire et suscite de nouvelles questions. De telles avancées dans ce domaine permettraient d'améliorer les pièges attractifs et représentent ainsi un défi majeur, étant donné la recrudescence de ce nuisible en Europe et sa résistance aux biocides couramment employés.

Mot-clés. Cimex, orientation, kairomones, substance sémiochimique, lutte anti-insecte, stimulus, relation hôte parasite.

Host-seeking behavior in the bed bug (Cimex lectularius) and applications in integrated pest management. A review Description of the subject. This study analyzes host-seeking behavior in the common bed bug, Cimex lectularius, paying particular attention to human stimuli involved in this orientation process. The potential applications in integrated pest management are discussed.

Literature. The common bed bug is an obligate hematophagous that has been associated with humans for millennia. When searching for food, this insect relies for orientation on signals produced by its hosts. Carbon dioxide emitted by human respiration is more attractive to bugs than body heat. The response of bed bugs to other volatile organic compounds released by the human body has been tested, but their perception and behavioral impact are not always studied together, and some discrepancies occasionally appear.

Conclusions. Currently, carbon dioxide is the most efficient lure for bed bugs, although real human bait is more attractive. Some home-made traps baited with dry ice are more efficient than other traps using complicated chemical blends. Dry ice seems to be more efficient as a lure than complex chemical blends, and it can be used in simple traps. Our knowledge of host-seeking behavior in bed bugs is still partial and new questions are constantly arising. Further efforts in the study of the chemical ecology of this process are needed in order to improve the management of this pest. The control of bed bugs in European countries appears to be a major challenge for the years to come.

Keywords. Cimex, orientation, kairomones, semiochemicals, insect control, stimuli, host parasite relations.

\section{INTRODUCTION}

Depuis la fin des années 1990, une recrudescence des populations de punaises de lit (Cimex lectularius L.)
(Hémiptères : Cimicidae) s'observe aux États-Unis (Anderson et al., 2008), en Australie (Doggett et al., 2004) et en Europe (Kilpinen et al., 2008). Ce fléau urbain touche principalement les secteurs hôteliers et 
hospitaliers sans épargner les habitations de particuliers (Doggett et al., 2012 ; Liu et al.,2015). Une fois installé, ce parasite exclusivement hématophage (Reinhardt et al., 2007) incommode les occupants par ses piqûres nocturnes pouvant entrainer des démangeaisons et des surinfections sévères (Thomas et al., 2004). La punaise de lit serait par ailleurs un vecteur potentiel d'agents infectieux tels que Trypanosoma cruzi, un parasite responsable de la maladie de Chagas (Salazar et al., 2014). Environ 45 pathogènes liés à l'Homme sont également capables de s'associer à la punaise de lit dont la bactérie Bartonella quintana (Leulmi et al., 2015), les virus de l'hépatite B, du sida (Goddard et al., 2009) et peut-être celui responsable de la maladie d'Ebola (Quarles, 2015a). Toutefois, il n'existe jusqu'à présent aucune étude démontrant la capacité de la punaise à transmettre ces pathogènes à l'Homme (Goddard et al., 2009 ; Quarles, 2015a).

L'efficacité des biocides employés pour lutter contre cet insecte diminue suite au développement de souches résistantes qui explique en majeure partie la résurgence observée (Romero et al., 2007). L'inconfort occasionné par cet insecte et la difficulté de l'éliminer incitent la société à adopter des comportements d'ostracisme (Hwang et al., 2005) pouvant à terme engendrer une crise dans les secteurs hôteliers et hospitaliers et nuire au tourisme (Eddy et al., 2011 ; Liu et al., 2015). Par ailleurs, les infestations ont des répercussions psychologiques et émotionnelles non négligeables sur les habitants (Goddard et al., 2012 ; Susser et al., 2012). Les symptômes observés (cauchemars, insomnie, anxiété, hyper vigilance, etc.) peuvent mener à terme à un trouble post-traumatique nécessitant une prise en charge psychologique (Goddard et al., 2012). La résurgence de la punaise de lit dans les pays développés représente donc une sérieuse menace économique et sanitaire (Eddy et al., 2011 ; Liu et al., 2015).

Les méthodes de lutte intégrée sont de plus en plus encouragées afin de limiter le développement de résistances aux biocides chimiques employés (Koganemaru et al., 2013). Les pièges adhésifs ou à fosse, utilisant des substances attractives pour la punaise de lit, sont ainsi des techniques de surveillance indispensables pouvant fortement contribuer à la diminution de l'infestation (Koganemaru et al., 2013 ; Vaidyanathan et al., 2013). Ces outils se basent le plus souvent sur l'utilisation de stimuli guidant l'insecte lors de sa recherche alimentaire (Koganemaru et al., 2013 ; Vaidyanathan et al., 2013). Or, beaucoup de désaccords subsistent sur les facteurs influençant ce comportement chez la punaise de lit (Siljander, 2006). Cette revue bibliographique présente la relation liant la punaise à l'Homme en portant une attention particulière au processus de recherche de l'hôte et aux stimuli impliqués. Il s'agit de faire le point sur les substances sémiochimiques émises par l'Homme et sur leur utilisation dans des techniques de surveillance et de contrôle des populations de punaises de lit.

\section{HÉMATOPHAGIE STRICTE ET ACTIVITÉ ALIMENTAIRE}

La punaise de lit est un parasite exclusivement hématophage (Reinhardt et al., 2007) fortement associé à l'Homme depuis des millénaires (Panagiotakopulu et al., 1999). Cet insecte montre une préférence pour le sang humain mais peut toutefois s'attaquer à d'autres animaux (e.g. chauve-souris, volatiles) lorsque son hôte privilégié fait défaut dans son environnement (Rivnay, 1932). En cas de famine, les punaises adultes survivraient de 4 à 18 mois sans repas (Polanco et al., 2011). Par ailleurs, les souches résistantes aux pyréthrinoides seraient plus sensibles aux effets de la famine (Polanco et al., 2011).

Les punaises de lit sont photophobes: elles se rassemblent pendant la journée avec leurs congénères au sein d'agrégats, à l'abri de la lumière, situés à proximité de sites de repos de l'Homme (Reis et al., 2011). Elles entrent en activité une fois la nuit tombée, bien que la présence d'un hôte précipite leur recherche alimentaire aussi bien au cours des phases nocturne (i.e. scotophase) que diurne (i.e. photophase) (Aak et al., 2014). Une fois l'hôte trouvé, les individus se gorgent de sang en 10 à 20 min (Reinhardt et al., 2007) et retournent aussitôt se mettre à l'abri au sein d'un agrégat (Reis et al., 2011). Tant qu'aucune prise alimentaire n'est concluante, les individus continuent leur recherche. Néanmoins, $2 \mathrm{~h}$ avant la photophase, la majorité des punaises rejoignent l'agrégat, qu'elles se soient ou non nourries (Reis et al., 2011).

Après un repas, l'activité des adultes décline, mais les femelles redeviennent sensibles à la présence de l'hôte plus rapidement. Elles sont davantage actives que les mâles et plus promptes à sortir de l'agrégat durant la photophase lorsqu'elles perçoivent les stimuli associés à la présence de l'hôte humain (Aak et al., 2014).

\section{PROCESSUS D'ORIENTATION VERS UN HÔTE}

\subsection{Généralités}

Afin de minimiser le temps passé hors de l'agrégat à rechercher leur hôte, les punaises de lit utilisent les stimuli chimiques émis par ce dernier. Certains de ces signaux permettent à la punaise de s'orienter depuis une position éloignée, alors que d'autres n'ont un rôle qu'une fois à proximité de l'hôte (Reinhardt et al., 2007). La distance à laquelle la punaise de lit 
est capable de détecter un hôte est encore incertaine. Certaines études évoquent une distance maximale de $150 \mathrm{~cm}$ (Anderson et al., 2009), alors que d'autres ont observé un comportement d'orientation dès $6 \mathrm{~m}$ (Usinger, 1966).

Chez les insectes, les sensilles permettent la perception des signaux présents dans leur environnement. Principalement situées sur les antennes et les pièces buccales, les sensilles de C.lectularius sont divisées en trois catégories : olfactives, gustatives et mécano-sensorielles. Ces organes sensoriels peuvent également être munis de récepteurs thermiques ou hydriques (Guidobaldi et al., 2014).

\subsection{Orientation thermique}

Les punaises de lit sont capables de détecter des différences de température de un ou deux degrés et sont attirées par toute source émettant une chaleur supérieure à celle de l'air ambiant. Cependant, au-delà de $43{ }^{\circ} \mathrm{C}$, la chaleur émise devient répulsive (Siljander, 2006). La distance à laquelle cette différence de température est perçue est encore incertaine (Siljander, 2006) mais d'après l'expérience de Singh et al. (2012), elle serait de $25 \mathrm{~cm}$.

Chez Cimex hemipterus, la punaise de lit tropicale, des récepteurs thermiques ont déjà été identifiés sur le pédicelle antennaire (Weeks et al., 2011). Des structures similaires ont été observées sur certaines sensilles antennaires de C.lectularius. Bien que leur rôle ne soit pas encore clairement démontré, les similarités morphologiques avec C.hemipterus laissent penser que ces sensilles ont également un rôle dans le thermotactisme chez les punaises de lit (Olson et al., 2014).

Si certains auteurs considéraient la chaleur comme le facteur influençant majoritairement la localisation de l'hôte (Siljander, 2006), ce constat semble être démenti par plusieurs études ayant observé le faible impact de ce stimuli comparé aux stimuli chimiques, notamment lors d'essais de piégeage (Anderson et al., 2009 ; Wang et al., 2009 ; Singh et al., 2012).

\subsection{Chimiotropisme}

Réponse comportementale. Les substances volatiles émises par l'Homme agissent fortement sur l'orientation de la punaise de lit, justifiant le nombre d'études qui leur sont consacrées (Harraca et al., 2012). Le dioxyde de carbone $\left(\mathrm{CO}_{2}\right)$ est une kairomone reconnue pour agir, seule ou en synergie avec d'autres composés, sur l'orientation des insectes hématophages vers leur hôte (Guerenstein et al., 2008). Il est considéré comme le stimulus ayant la plus grande influence sur le comportement de recherche d'hôte chez les punaises de lit (Anderson et al., 2009; Wang et al., 2009;
Aak et al., 2014). Ce composé volatil serait l'indice le plus représentatif de la présence d'un hôte puisque le remplacement de ce dernier par une source de $\mathrm{CO}_{2}$ engendre chez $C$. lectularius une agrégation rapide vers la source artificielle de $\mathrm{CO}_{2}$ (Aak et al., 2014). L'effet attractant du $\mathrm{CO}_{2}$ serait indépendant du débit de son émission (Singh et al., 2012) mais amélioré par l'ajout d'acide lactique (Wang et al., 2009) ou d'un mélange de nonanal, d'1-octen-3-ol, d'huile de menthe verte et d'huile de coriandre égyptienne (Singh et al., 2012).

La perception antennaire et l'activité comportementale de plusieurs autres composés volatils émis par l'Homme, seuls ou en mélange, ont été investiguées (Tableau 1). Bien que les résultats obtenus pour des composés testés individuellement apportent une base d'information, ceux-ci ne sont pas représentatifs d'une situation réelle où de nombreuses substances sémiochimiques pourraient agir en synergie. De plus, il est courant qu'un composé isolé n'engendre aucune réponse comportementale, mais soit néanmoins impliqué dans le processus de localisation de l'hôte, lorsqu'il est associé à d'autres molécules sémiochimiques. Par exemple, Singh et al. (2012) ont démontré qu'un mélange d'huile de coriandre égyptienne, d'huile de menthe verte, d'1-octen-3-ol et de nonanal a une meilleure attractivité vis-à-vis de C. lectularius que chaque composé employé seul.

Les tests comportementaux basés sur un mélange de kairomones sont pour leur part limités quant à l'interprétation du degré d'implication de chaque composé dans la réponse de l'insecte. De plus, les proportions des composants du mélange peuvent également jouer un rôle dans la réponse induite (Weeks et al., 2011). Par exemple, Harraca et al. (2012) ont récolté les molécules volatiles corporelles de 24 volontaires et ont analysé l'influence du ratio en sulcatone et aldéhydes comportant de 7 à 10 atomes de carbone. Ils constatent que les punaises s'activent davantage à chercher un hôte lorsque le ratio entre ces composés est faible.

Réponse électrophysiologique. Les conclusions tirées des études comportementales reposent sur la condition indispensable que les substances testées soient perçues physiologiquement par l'insecte. Or, les études comportementales ne démontrent pas toujours la réponse électrophysiologique au niveau des antennes de l'insecte, voire, dans certains cas, sont en contradiction avec de précédentes études l'analysant (Tableau 1).

Chez la punaise de lit, les sensilles olfactives se trouvent uniquement au niveau des antennes (Steinbrecht et al., 1976). Deux régions olfactives (RO) ont déjà été identifiées : elles se trouvent toutes les deux sur le segment terminal du flagelle, l'une étant sur la face interne (ROI) et l'autre sur la face externe (ROII) 
Tableau 1. Références étudiant l'impact électrophysiologique et/ou comportemental de composés volatils émis par l'Homme chez la punaise de lit - References studying electrophysiological and/or behavioural impacts of human volatile compounds on bed bugs

\begin{tabular}{|c|c|c|c|c|c|}
\hline \multirow[t]{2}{*}{ Composé volatil } & \multicolumn{3}{|c|}{ Impact électrophysiologique } & \multicolumn{2}{|c|}{ Impact comportemental } \\
\hline & Référence & Effet & Sensilles antennaires & Référence & Effet \\
\hline Acide butyrique & Harraca et al., 2010 & - & & Anderson et al., 2009 & +1 \\
\hline Acide propionique & & & & Anderson et al., 2009 & $+{ }^{1}$ \\
\hline Acide valérique & & & & Anderson et al., 2009 & $+{ }^{1}$ \\
\hline Acide L-lactique & Harraca et al., 2010 & - & & $\begin{array}{l}\text { Anderson et al., } 2009 \\
\text { Wang et al., } 2009 \\
\text { Singh et al., } 2012\end{array}$ & $\begin{array}{l}+^{1} \\
-2 \\
+^{3}\end{array}$ \\
\hline 1-Octen-3-ol & Harraca et al., 2010 & - & & $\begin{array}{l}\text { Anderson et al., } 2009 \\
\text { Wang et al., } 2009 \\
\text { Singh et al., } 2012\end{array}$ & $\begin{array}{l}+^{1} \\
-2 \\
+^{3},+^{4}\end{array}$ \\
\hline R-Octénol & & & & Singh et al., 2012 & -5 \\
\hline Heptanal & Harraca et al., 2012 & + & $\mathrm{Db}, \mathrm{Dg}$ & Harraca et al., 2012 & $+^{6}$ \\
\hline Octanal & Harraca et al., 2012 & + & $\mathrm{Db}, \mathrm{Dg}$ & Harraca et al., 2012 & $+^{6}$ \\
\hline Nonanal & Harraca et al., 2012 & + & $\mathrm{Db}, \mathrm{Dg}$ & $\begin{array}{l}\text { Harraca et al., } 2012 \\
\text { Siljander et al., } 2008 \\
\text { Singh et al., } 2012\end{array}$ & $\begin{array}{l}+^{6} \\
+^{7} \\
+^{3},+^{4}\end{array}$ \\
\hline Décanal & Harraca et al., 2012 & + & $\mathrm{Db}, \mathrm{Dg}$ & $\begin{array}{l}\text { Harraca et al., } 2012 \\
\text { Siljander et al., } 2008\end{array}$ & $\begin{array}{l}+^{6} \\
+^{7}\end{array}$ \\
\hline Nonanal & Harraca et al., 2012 & + & $\mathrm{Db}, \mathrm{Dg}$ & $\begin{array}{l}\text { Harraca et al., } 2012 \\
\text { Singh et al., } 2012 \\
\text { Siljander et al., } 2008\end{array}$ & $\begin{array}{l}+^{6} \\
+^{3} \\
+^{4},+^{7}\end{array}$ \\
\hline Sulcatone & $\begin{array}{l}\text { Harraca et al., } 2010 \\
\text { Harraca et al., } 2012\end{array}$ & $\begin{array}{l}+ \\
+\end{array}$ & $\begin{array}{l}\mathrm{Dg} \\
\mathrm{Db}\end{array}$ & $\begin{array}{l}\text { Harraca et al., } 2012 \\
\text { Siljander et al., } 2008 \\
\text { Singh et al., } 2012\end{array}$ & $\begin{array}{l}+^{6} \\
+{ }^{7} \\
-3\end{array}$ \\
\hline L-carvone & & & & Singh et al., 2012 & $+^{3}$ \\
\hline Géranyl acétone & & & & Singh et al., 2012 & -3 \\
\hline Indole & Harraca et al., 2010 & + & $\mathrm{Dg}$ & & \\
\hline $\mathrm{NH}_{3} \mathrm{HCO}_{3}$ & & & & Singh et al., 2012 & -5 \\
\hline Diméthyl trisulfide & Harraca et al., 2010 & + & $\mathrm{Db}$ & Gries et al., 2015 & $+^{8}$ \\
\hline Ammoniac & Harraca et al,. 2010 & + & $\mathrm{C}$ & & \\
\hline Acétone & Harraca et al., 2010 & - & & & \\
\hline E-2-octénal & Harraca et al., 2010 & + & $\mathrm{Db}$ & $\begin{array}{l}\text { Gries et al., } 2015 \\
\text { Siljander et al., } 2008\end{array}$ & $\begin{array}{l}+^{8} \\
+^{7}\end{array}$ \\
\hline Benzaldéhyde & Harraca et al., 2010 & - & & Siljander et al., 2008 & $+^{7}$ \\
\hline Butanone & Harraca et al., 2010 & - & & & \\
\hline Diméthyl disulfide & Harraca et al., 2010 & - & & Gries et al., 2015 & $+^{8}$ \\
\hline Limonène & Harraca et al., 2010 & - & & Siljander et al., 2008 & $+^{7}$ \\
\hline Phénol & Harraca et al., 2010 & - & & & \\
\hline
\end{tabular}

Effet positif (+) ou négatif (-) observé pour : ${ }^{1}$ : un mélange d'acide propionique, d'acide butyrique, d'acide valérique, d'1-octen-3-ol et d'acide L-lactique $;{ }^{2}$ : un mélange d'1-octen-3-ol et d'acide L-lactique $;{ }^{3}:$ un composé volatil testé seul $;{ }^{4}:$ un mélange de nonanal, d'1-octen-3-ol, d'huile de menthe verte et d'huile de coriandre égyptienne $;{ }^{5}$ : un mélange de R-octénol et d' $\mathrm{NH}_{3} \mathrm{HCO}_{3} ;{ }^{6}$ : un extrait d'odeur corporelle humaine $;{ }^{7}$ : un mélange de nonanal, décanal, (E)-2-hexénal, (E)-2-octénal, (2E,4E)-octadiénal, benzaldéhyde, (+)- et (-)-limonène, sulcatone et d'alcool benzylique ; ${ }^{8}$ : un mélange de diméthyl disulfide, diméthyl trisulfide, (E)-2-hexénal, (E)-2-octénal et 2-hexanone - Positive effect $(+)$ or negative effect (-) induced by: ${ }^{1}:$ a blend of propionic acid, butyric acid, valeric acid, 1 -octen-3-ol and lactic acid $;{ }^{2}:$ a blend of 1 -octen-3-ol and lactic acid; ${ }^{3}:$ volatile compound tested alone; ${ }^{4}$ : a blend of nonanal, 1-octen-3-ol, spearmint oil and coriander Egyptian oil; ${ }^{5}:$ a blend of R-octenol and $\mathrm{NH}_{3} \mathrm{HCO}_{3} ;{ }^{6}:$ human odour extract; ${ }^{7}:$ a blend of nonal, decanal, (E)-2-hexenal, (E)-2-octenal , (2E,4E)-octadienal, benzaldehyde, (+)-and (-)-limonene, sulcatone and benzyl alcohol; ${ }^{8}:$ a blend of dimethyl disulphide, dimethyl trisulfide, (E)-2-hexenal, (E)-2-octenal and 2-hexanone. 
(Levinson et al., 1974 ; Steinbrecht et al., 1976). Sept types de sensilles existeraient dont celles nommées C, $\mathrm{D}$ et $\mathrm{E}$ ayant un rôle olfactif (Levinson et al., 1974 ; Steinbrecht et al., 1976). La région olfactive interne comprend six sensilles de type D qui sont divisées en trois groupes distincts (i.e. $\mathrm{D} \alpha, \mathrm{D} \beta$ et $\mathrm{D} \gamma$ ) (Harraca et al., 2010). Neuf sensilles de type $C$ sont réparties sur la ROI (4) et la ROII (5). Environ 29 sensilles de type E sont réparties sur ces deux régions principalement et sont divisées en deux groupes (i.e. E1et E2). Ainsi, le système olfactif de la punaise de lit est constitué de 44 sensilles antennaires présentes principalement sur le segment terminal du flagelle (Levinson et al., 1974 ; Steinbrecht et al., 1976). Leur répartition reste équivalente entre les individus (Harraca et al., 2010) et ne présente pas de dimorphisme sexuel mais diffère légèrement chez les nymphes de premier stade (Levinson et al., 1974 ; Steinbrecht et al., 1976).

\subsection{Orientation tactile et visuelle}

L'orientation de la punaise de lit via des stimuli tactiles ou visuels est encore très peu documentée (Singh et al., 2015). L'antenne de cet insecte dispose de récepteurs mécaniques lui permettant de percevoir les reliefs du terrain (Steinbrecht et al., 1976). Les punaises de lit préfèrent se déplacer sur des surfaces à la texture légèrement rugueuse, celles trop lisses l'empêchant de grimper et celles trop rugueuses (e.g. feutre) l'empêtrant dans ses mouvements (Singh et al., 2015). Dans une pièce, les punaises de lit s'orientent préférentiellement vers les éléments verticaux pouvant faire penser aux pieds du mobilier. Les supports de couleur foncée (noire, brune ou rouge) sont également préférés, sans doute à cause de la photosensibilité de l'insecte (Singh et al., 2015).

\section{LA LUTTE EN EUROPE}

\subsection{Problématique des biocides}

L'utilisation de biocides est de plus en plus remise en question suite à la capacité de C. lectularius à y résister (Koganemaru et al., 2013). Depuis quelques années, ce nuisible montre une résistance contre les pyréthrinoïdes qui représentent la classe de pesticides la plus communément employée (Romero et al., 2007). Pour pallier cette résistance, de nouvelles formulations combinent des néonicotinoïdes et pyréthrinoïdes. Cependant, l'apparition d'une nouvelle résistance est à craindre (Potter et al., 2012 ; Gordon et al., 2014).

Face à cette problématique, de nouveaux produits chimiques alternatifs sont investigués. Le potentiel biocide du champignon entomopathogène Beauveria bassiana montre des résultats encourageants dans la lutte contre la punaise de lit: en laboratoire, une exposition directe de $50 \%$ de la population à ce champignon engendre $95 \%$ de mortalité (Barbarin et al., 2012). Les huiles essentielles de cèdre, de clou de girofle ou de menthe poivrée semblent présenter des activités insecticides sur les punaises de lit. Leur application nécessite toutefois une pulvérisation directe sur l'insecte, ce qui peut s'avérer difficile en pratique (Singh et al., 2014).

\subsection{Détection et interception}

Quelques individus suffisent pour démarrer un nouveau foyer d'infestation (Fountain et al., 2014 ; Akhoundi et al., 2015), d'autant plus que les femelles gravides sont les plus enclines à s'éloigner de l'agrégat (Pfiester et al., 2009). Parmi les méthodes de lutte intégrée, les outils de détection et de piégeages (Tableau 2) sont les plus conseillés, que ce soit pour évaluer la progression d'une infestation ou limiter les risques d'une flambée épidémique (e.g. Ville de New York, 2009-2010) (Koganemaru et al., 2013 ; Vaidyanathan et al., 2013). Il peut s'agir de systèmes mettant en évidence la présence de punaises (i.e. via ses fèces) ou de pièges les interceptant, qui permettent en même temps de réduire le nombre d'individus. Ces appareils de piégeage sont répartis en deux groupes : les pièges passifs et actifs. Les méthodes passives consistent généralement en des pièges à fosse ou englués n'utilisant aucun leurre : ces pièges se limitent aux aires de repos de la punaise de lit ou se placent sur son parcours afin de l'intercepter. Elles nécessitent une bonne connaissance des endroits propices à l'installation de l'insecte et doivent anticiper le déplacement des individus (Vaidyanathan et al., 2013). À l'inverse de ces techniques de piégeage passif, celles actives utilisent des leurres attirant l'insecte. Les substances chimiques employées par ces appareils ne sont pas toujours dévoilées par les fournisseurs (Vaidyanathan et al., 2013).

Ces outils de piégeage souffrent de limites. Dans le cas des pièges passifs, leur efficacité dépend du degré d'activité locomotrice de l'insecte, qui varie suivant le stade de développement, le sexe et le statut nutritionnel. La présence d'un être humain améliore fortement l'efficacité de ces pièges mais l'expose à des piqûres (Koganemaru et al., 2013 ; Vaidyanathan et al., 2013).

La majorité des méthodes d'interception actives utilise des stimuli imitant ceux émis par l'Homme. Ces pièges ciblent donc principalement les individus lancés à la recherche d'un hôte et donc affamés (Vaidyanathan et al., 2013) puisqu'une fois nourris, les signaux émis par l'hôte n'ont plus autant d'effet et peuvent même devenir répulsifs (Reinhardt et al., 2007). De plus, Anderson et al. (2009) ont mis en évidence que les différents stades ne répondent pas forcément de la même 
Tableau 2. Principes passifs et actifs de différents systèmes de détection et d'interception de punaises de lit - Active or passive functioning principles of different devices for bed bug detection or catching.

\begin{tabular}{|c|c|c|c|}
\hline \multicolumn{2}{|c|}{ Principe passif ou actif } & \multirow{2}{*}{$\begin{array}{l}\text { Type d'appareil } \\
\text { Support adhésif }\end{array}$} & \multirow{2}{*}{$\begin{array}{l}\text { Source internet } \\
\text { www.domyownpestcontrol.com } \\
\text { www.edialux.fr } \\
\text { www.buggybeds.com }\end{array}$} \\
\hline Passif & À placer à des endroits de passage stratégiques & & \\
\hline Passif & Simule une zone de refuge & Support adhésif & www.sternenvironmental.com \\
\hline Passif & À placer aux pieds du lit & Piège à fosse & $\begin{array}{l}\text { www.insect-interceptor.com } \\
\text { www.bedmoat.com }\end{array}$ \\
\hline Passif & Mise en évidence des fèces & Kit de réactifs & www.sternenvironmental.com \\
\hline Actif & Émission de chaleur & $\begin{array}{l}\text { Piège à fosse } \\
\text { avec support } \\
\text { adhésif }\end{array}$ & www.sternenvironmental.com \\
\hline Actif & Émission de $\mathrm{CO}_{2}$ et utilisation d'une huile minérale & Piège à fosse & www.sternenvironmental.com \\
\hline Actif & $\begin{array}{l}\text { Émission de } \mathrm{CO}_{2} \text { et d'une kairomone (attraction des } \\
\text { individus cherchant un hôte) et d'une phéromone } \\
\text { d'agrégation (attraction des individus cherchant à s'agréger) }\end{array}$ & $\begin{array}{l}\text { Piège à fosse et } \\
\text { mise en évidence } \\
\text { des fèces }\end{array}$ & www.fmcprosolutions.com \\
\hline Actif & Émission de chaleur, de $\mathrm{CO}_{2}$ et d'humidité & Support adhésif & www.bbalertactive.com \\
\hline Actif & $\begin{array}{l}\text { Émission d'une chaleur, de } \mathrm{CO}_{2} \text { et d'une phéromone } \\
\text { (SpringStar) imitant la sueur humaine }\end{array}$ & Support adhésif & www.edialux.fr \\
\hline Actif & Émission de chaleur, de $\mathrm{CO}_{2}$ et d'une kairomone & Piège à fosse & www.biosensory.com \\
\hline Actif & $\begin{array}{l}\text { Utilisation d'un mélange attractif alimentaire efficace sur } \\
\text { tous les stades de développement }\end{array}$ & Support adhésif & www.domyownpestcontrol.com \\
\hline Actif & Comprimés attractifs & Support adhésif & www.kapo.com/kapo-vert \\
\hline
\end{tabular}

manière aux différents leurres employés. Par ailleurs, dans certains cas, les substances chimiques employées ne semblent pas avoir été préalablement testées pour en certifier la perception et le rôle comportemental chez la punaise de lit (Weeks et al., 2011 ; Koganemaru et al., 2013).

Les pièges combinant du $\mathrm{CO}_{2}$ à un leurre chimique ou thermique sont tout aussi efficaces que ceux utilisant les trois artifices en même temps (Anderson et al., 2009 ; Singh et al., 2012). Des pièges artisanaux à base de glace carbonique seraient même plus efficaces que des pièges plus complexes (Wang et al., 2011).

\section{CONCLUSIONS}

La résurgence des punaises de lit est un problème sanitaire et économique auquel l'Europe fait face (Boase, 2001 ; Kilpinen et al., 2008 ; Bencheton et al., 2011). La capacité de cet insecte à développer une résistance face aux biocides actuellement disponibles accroît la nécessité de développer des méthodes de lutte intégrée. Ces nouvelles mesures se baseraient sur des traitements mécaniques couplés à des outils de prévention et de détection précoce (Quarles, 2015b). Parmi ces moyens de détection, l'inspection canine est une technique devenant de plus en plus populaire
(Vaidyanathan et al., 2013). Pourtant, son efficacité sur le terrain serait remise en doute (Cooper et al., 2014) et présenterait même un risque de propager l'infestation (Vaidyanathan et al., 2013). Les appareils de détection actifs basés sur des leurres attractifs permettent une meilleure estimation de l'infestation par rapport aux inspections visuelles et à la plupart des méthodes passives (Anderson et al., 2009; Wang et al., 2009 ; Wang et al., 2011 ; Koganemaru et al., 2013; Vaidyanathan et al., 2013). Cependant, ils ciblent préférentiellement les individus affamés. Il convient donc dans certains cas de laisser la pièce vide pendant plusieurs jours afin que le piège soit plus efficace (Koganemaru et al., 2013). Il serait intéressant d'étudier l'efficacité des leurres chimiques selon le statut nutritionnel de l'insecte.

Par ailleurs, les leurres utilisés restent moins efficaces qu'un réel appât humain (Koganemaru et al., 2013). Il est donc important de trouver des leurres simulant la présence d'un hôte afin d'attirer les insectes dans les pièges sans exposer un être humain à des risques (Weeks et al., 2011). Jusqu'à ce jour, le $\mathrm{CO}_{2}$ semble être le leurre le plus efficace (Anderson et al., 2009 ; Wang et al., 2009 ; Wang et al., 2011 ; Koganemaru et al., 2013 ; Vaidyanathan et al., 2013).

Anderson et al. (2009) ont montré que les pièges attractifs attrapent autant de punaises de lit de stade 1 et 
2 que les pièges sans appât. Les futures études analysant l'efficacité des molécules attractives devraient considérer le stade des individus ciblés. Ceci permettrait de mettre au point des pièges interceptant davantage les jeunes stades et d'enrayer ainsi le tout début d'une infestation. Il serait également intéressant d'étudier l'impact des substances volatiles testées selon leur gradient de dispersion dans l'air ambiant: il est possible qu'une fois à proximité d'un piège, l'insecte perçoive les composés à trop forte concentration et soit finalement repoussé. Intégrer ces nouveaux aspects dans les études à venir pourrait permettre de mieux comprendre le processus d'orientation de la punaise vers son hôte et d'améliorer les pièges attractifs.

L'influence de l'odeur humaine sur l'attraction des insectes hématophages est un domaine actuellement porteur. Cependant, peu d'études s'intéressent au rôle du microbiome humain sur l'attraction des punaises de lit, alors que son influence sur la composition des substances volatiles humaines émises est déjà avérée (Dormont et al., 2013). Verhulst et al. (2011) ont déjà vérifié l'influence du microbiome cutané sur l'attraction d'Anopheles gambiae, un moustique vecteur de la malaria. Il est également probable que ce microbiome influence l'attraction de la punaise. Ceci pourrait expliquer la forte sélectivité de cet insecte vis-à-vis de l'Homme à l'inverse de la température corporelle et de l'émission de $\mathrm{CO}_{2}$ qui sont des points communs à tous les vertébrés.

Ainsi, beaucoup d'investigations doivent encore être menées afin d'améliorer notre compréhension du processus d'attraction de la punaise de lit vis-à-vis de l'Homme. De telles avancées dans ce domaine offriraient de nouvelles perspectives et répondraient ainsi à une demande actuelle mondiale pour de nouvelles solutions efficaces contre la punaise de lit.

\section{Bibliographie}

Aak A. et al., 2014. Questing activity in bed bug populations: male and female responses to host signals. Physiol. Entomol., 39(3), 199-207.

Akhoundi M. et al., 2015. Spatial genetic structure and restricted gene flow in bed bugs (Cimex lectularius) populations in France. Infect. Genet. Evol., 34, 236-243.

Anderson A.L. \& Leffler K., 2008. Bedbug infestations in the news: a picture of an emerging public health problem in the United States. J. Environ. Health, 70(9), 24-27.

Anderson J.F. et al., 2009. A carbon dioxide, heat and chemical lure trap for the bedbug, Cimex lectularius. Med. Vet. Entomol., 23(2), 99-105.

Barbarin A.M. et al., 2012. A preliminary evaluation of the potential of Beauveria bassiana for bed bug control. J. Invertebrate Pathol., 111, 82-85.

Bencheton A.L. et al., 2011. Resurgence of bedbugs in southern France: a local problem or the tip of the iceberg? J. Eur. Acad. Dermatol. Venereology, 25, 599602.

Boase C.J., 2001. Public health bedbugs - back from the brink. Pestic. Outlook, 12, 159-162.

Cooper R. et al., 2014. Accuracy of trained canines for detecting bed bugs (Hemiptera: Cimicidae). J. Econ. Entomol., 107(6), 2171-2181.

Doggett S.L., Geary M.J. \& Russell R.C., 2004. The resurgence of bed bugs in Australia: with notes on their ecology and control. Environ. Health, 4(2), 30-38.

Doggett S.L. et al., 2012. Bed bugs: clinical relevance and control options. Clin. Microbiol. Rev., 25(1), 164-192.

Dormont L., Bessière J.M. \& Cohuet A., 2013. Human skin volatiles: a review. J.Chem. Ecol., 39, 569-578.

Eddy C. \& Jones S.C., 2011. Bed bugs, public health, and social justice: part 1, a call to action. J. Environ. Health, 73, 8-14.

Fountain T. et al., 2014. Human-facilitated metapopulation dynamics in an emerging pest species, Cimex lectularius. Mol.Ecol., 23(5), 1071-1084.

Goddard J. \& de Shazo R., 2009. Bed bugs (Cimex lectularius) and clinical consequences of their bites. J. Am. Med. Assoc., 301(13), 1358-1366.

Goddard J. \& de Shazo R., 2012. Psychological effects of bed bug attacks (Cimex lectularius L.). Am. J. Med., 125(1), 101-103.

Gordon J.R. et al., 2014. Trouble brewing for insecticides? Pest Control Technol., 42(6), 72-80.

Gries R. et al., 2015. Bed bug aggregation pheromone finally identified. Angew. Chem. Int. Ed., 54, 1135-1138.

Guerenstein P.G. \& Hildebrand J.G., 2008. Roles and effects of environmental carbon dioxide in insect life. Аппи. Rev. Entomol., 53, 161-178.

Guidobaldi F., May-Concha I.J. \& Guerenstein P.G., 2014. Morphology and physiology of the olfactory system of blood-feeding insects. J. Physiol. Paris, 108, 96-111.

Harraca V. et al., 2010. Characterization of the antennal olfactory system of the bed bug (Cimex lectularius). Chem. Senses, 35(3), 195-204.

Harraca V. et al., 2012. Smelling your way to food: can bed bugs use our odour? J. Exp. Biol., 215, 623-629.

Hwang S.W. et al., 2005. Bed bug infestations in an urban environment. Emerging Infect. Dis., 11(4), 533-538.

Kilpinen O., Jensen K.-M.V. \& Kristensen M., 2008. Bed bug problems in Denmark, with a European perspective. In: Robinson W.H. \& Bajomi D., eds. Proceedings of the $6^{\text {th }}$ International conference on urban pests, 13-16 July 2008, Budapest, Hungary. Veszprem, Hungary: OOKPress Kft, 395-399.

Koganemaru R. \& Miller D.M., 2013. The bed bug problem: past, present, and future control methods. Pestic. Biochem. Physiol., 106(3), 177-189.

Leulmi H. et al., 2015. Competence of Cimex lectularius bed bugs for the transmission of Bartonella quintana, the agent of trench fever. PLoS Negl. Trop. Dis., 9(5), e0003789. 
Levinson H.Z. et al., 1974. Structure of sensilla, olfactory perception, and behaviour of bedbug, Cimex lectularius, in response to its alarm pheromone. J. Insect Physiol., 20, 1231-1248.

Liu B. \& Pennington-Gray L., 2015. Bed bugs bite the hospitality industry? A framing analysis of bed bug news coverage. Tourism Manage., 48, 33-42.

Olson J.F. et al., 2014. Morphology, ultrastructure and functional role of antennal sensilla in off-host aggregation by the bed bug, Cimex lectularius. Arthropod Struct. Dev., 43(2), 117-122.

Panagiotakopulu E. \& Buckland P.C., 1999. Cimex lectularius L., the common bed bug from pharaonic Egypt. Antiquity, 73, 908-911.

Pfiester M., Koehler P.G. \& Pereira R.M., 2009. Effect of population structure and size on aggregation behavior of Cimex lectularius (Hemiptera: Cimicidae). J. Med. Entomol., 46(5), 1015-1020.

Polanco A.M., Miller D.M. \& Brewster C.C., 2011. Survivorship during starvation for Cimex lectularius L. Insects, 2, 232-242.

Potter M.F. et al., 2012. Dual-action bed bug killers. Pest Control Technol., 40, 62-76.

Quarles W., 2015a. Can bed bugs carry Ebola? IPM Pract., 34, 10-11.

Quarles W., 2015b. New IPM methods for bed bugs. IPM Pract., 34, 1-9.

Reinhardt K. \& Siva-Jothy M.T., 2007. Biology of the bed bugs (Cimicidae). Annu. Rev. Entomol., 52, 351-374.

Reis M.D. \& Miller D.M., 2011. Host searching and aggregation activity of recently fed and unfed bed bugs (Cimex lectularius L.). Insects, 2, 186-194.

Rivnay E., 1932. Studies in tropisms of the bed bug Cimex lectularius L. Parasitology, 24, 121-136.

Romero A. et al., 2007. Insecticide resistance in the bed bug: a factor in the pest's sudden resurgence? J. Med. Entomol., 44, 175-178.

Salazar R. et al., 2014. Bed bugs (Cimex lectularius) as vectors of Trypanosoma cruzi. Am. J. Trop. Med. Hyg., 92(2), 331-335.

Siljander E., 2006. Foraging and communication ecology of bed bugs, Cimex lectularius L. (Hemiptera: Cimicidae). Am. Entomol., 52, 116-117.
Siljander E. et al., 2008. Identification of the airborne aggregation pheromone of the common bed bug, Cimex lectularius. J. Chem. Ecol., 34, 708-718.

Singh N. et al., 2012. Interactions among carbon dioxide, heat, and chemical lures in attracting the bed bug, Cimex lectularius L. (Hemiptera: Cimicidae). Psyche, 2012, 1-9.

Singh N. \& Wang C., 2014. Potential of essential oil-based pesticides and detergents for bed bug control. J. Econ. Entomol., 107(6), 2163-2170.

Singh N., Wang C. \& Cooper R., 2015. Role of vision and mechanoreception in bed bug, Cimex lectularius L. behavior. PLoS One, 10(3), e0118855.

Steinbrecht R.A. \& Muller B., 1976. Fine structure of antennal receptors of bed bug, Cimex lectularius L. Tissue Cell, 8, 615-636.

Susser S.R. et al., 2012. Mental health effects from urban bed bug infestation (Cimex lectularius L.): a crosssectional study. BMJ Open, 2, e000838.

Thomas I., Kihiczak G.C. \& Schwartz R.A., 2004. Bedbug bites: a review. Int. J. Dermatol., 43, 430-433.

Usinger R.L., 1966. Monograph of Cimicidae (HemipteraHeteroptera). Thomas Say Foundation, 7. College Park, MD, USA: Entomological Society of America.

Vaidyanathan R. \& Feldlaufer M.F., 2013. Bed bug detection: current technologies and future directions. Am. J. Trop. Med. Hyg., 88(4), 619-625.

Verhulst N.O. et al., 2011. Composition of human skin microbiota affects attractiveness to malaria mosquitoes. PLoS One, 6(12), e28991.

Wang C. et al., 2009. Bed bug (Heteroptera: Cimicidae) attraction to pitfall traps baited with carbon dioxide, heat, and chemical lure. J. Econ. Entomol., 102(4), 1580-1585.

Wang C. et al., 2011. Effectiveness of bed bug monitors for detecting and trapping bed bugs in apartments. J. Econ. Entomol., 104, 274-278.

Weeks E.N.I. et al., 2011. Semiochemicals of the common bed bug, Cimex lectularius L. (Hemiptera: Cimicidae), and their potential for use in monitoring and control. Pest Manage. Sci., 67(1), 10-20.

(53 réf.) 